\title{
Estado actual de la odontopediatría en la República Mexicana
}

Enrique E.-Huitzil-Muñóz ${ }^{1}$

Samantha Abril-García-Damián ${ }^{2}$

María Guadalupe-Sol-Gamboa ${ }^{3}$

\section{Resumen}

La odontología pediátrica satisface un propósito muy especial con respecto de las especialidades odontológicas orientada a la prevención y curación de las enfermedades del sistema estomatognático durante la infancia y considerada como una especialidad muy completa y relativamente nueva se realiza una revisión acerca de la historia de la odontología pedíatrica en el mundo y en especial en México, recopilando información importante acerca de las instituciones universitarias que cuentan en su curricula con la especialidad, certificación y zonas de ubicación en la República Mexicana. Conclusiones: De acuerdo a las regiones de la República Mexicana en las que se encuentran ubicados los diversos posgrados, se concluye que de un total de 30 universidades que cuentan con la especialidad o maestría en odontopediatría, existen 7 en la denominada región centro y golfo y 6 en la región México D.F., estos datos concuerdan con el elevado número de habitantes que habitan esas regiones y que necesitan atención odontopediátrica cubriendo así la alta demanda del servicio por parte de los odontólogos posgraduados egresados.

Palabras clave: odontología pediátrica, certificación.

Artigo de revisão

\section{Situação atual da Odontopediatria no México}

\section{Resumo}

O objetivo deste artigo é informar aos egressos do curso de odontologia, odontopediatras e instituições encarregadas de certificar a qualidade de pós-graduação, sobre a distribuição das universidades que oferecem especialização ou mestrado em Odontopediatria e seu status de certificação. Realizou-se uma revisão a respei- to da história da Odontopediatria no mundo e em especial no México. Reuniu-se informação importante a respeito das instituições universitárias que oferecem a especialidade, seu status de certificação e sua localização na República Mexicana. Considerando as regiões da República Mexicana, de um total de 30 universidades que oferecem especialização ou mestrado em Odontopediatria, 7 estão na região denomina-

\footnotetext{
${ }^{1}$ Maestro en Ciencias estomatológicas en pediatría. Docente BUAP. E-mail: hutizilnet@yahoo.com

${ }^{2}$ Estudiante de 3 er año de la Maestría en Ciencias estomatológicas en pediatría. BUAP. E-mail: sabrilgd@hotmail.com

${ }^{3}$ Estudiante de 3er año de la Maestría en Ciencias estomatológicas en pediatría. BUAP. E-mail: luz_sol@hotmail.com
} 
da centro e golfo e 6 na região do Distrito Federal (D.F México). Conclusões: Estes dados são coerentes com o elevado número de habitantes dessas regiões, mas não com o número de população infantil que requerem os serviços odontopediátricos, já que nos estados com maior po- pulação de 0 a 14 anos são Chiapas e Guerreiro lugares onde, pelo baixo desenvolvimento em educação superior, não existem pós-graduações voltadas para a formação de odontopediatras.

Palavras chaves: odontopediatria, certificação.

Review article

\section{Current state in pediatric dentistry in México}

\section{Abstrac}

Pediatric dentistry meets a very special purpose in respect of the dental specialties focused on prevention and cure of diseases of the stomatognathic system during childhood and considered a specialty very complete and relatively new is a review about the history of dentistry pediatric in the world and especially in Mexico, gathering important information about the universities that have in their curriculum with specialty certification and location areas in Mexico. Conclusions: According to the regions of Mexico in which are located the various graduate programs, it is concluded that a total of 30 universities that offer specialty or expertise in dentistry, there are 7 in the so-called Gulf and central and 6 in the Mexico City region, these data are consistent with the high number of people living in these regions and in need of care odontopediátrica thus covering the high demand for the service by postgraduate dental graduates.

Keywords: pediatric dentistry, certification.

\section{Introducción}

Odontopediatría, pedodoncia, paidodoncia, estomatología infantil y odontología infantil son los términos más comunes utilizados al referirse al área de conocimiento que estudia la boca del niño y sus enfermedades. Así como la pediatría ocupa una posición singular en relación con las especialidades médicas, del mismo modo la odontología pediátrica satisface un propósito muy especial con respecto de las especialidades odontológicas, en cuanto a que está orientada a la prevención y curación de las enfermedades del sistema estomatognático durante la infancia.

Con el objetivo de satisfacer las necesidades en cuanto a salud bucal infantil se refiere, se requiere la formación de especialistas en el área, por ello es importante tener al alcance una recopilación de las instituciones universitarias con posgrados en odontología pediátrica reconocidas en nuestro país para poder así acercarnos a una estimación mas exacta sobre si la cobertura es proporcional a la demanda.

\section{Inicios de la asistencia dental infantil}

A finales del siglo XVIII se encuentran referencias de una asistencia dental en la infancia de una manera reglada. Más tarde la British Dental Association recomendó la odontología escolar obligatoria. 
En Estrasburgo, Francia, en 1902, se pone en funcionamiento la primera clínica municipal exclusivamente para niños, anexa a la Policlínica Universitaria bajo la dirección de Ernesto Jessen. En Estados Unidos hacia 1901, en Nueva York, se establece la primera clínica de asistencia odontopediátrica por los miembros de la Rochester Dental Society. George Eastman el hombre más rico e influyente de la ciudad aportó los fondos suficientes y creó en 1905 una gran clínica de asistencia dental infantil que se convirtió en un centro avanzado de formación de jóvenes licenciados especializados en odontología infantil. En 1913 se crea la primera sociedad científica dedicada a la odontología infantil. Fue en Detroit donde catorce dentistas formaron el Pedodontic Study Club con el objetivo de perfeccionar los conocimientos en el tratamiento dental. En 1925 se transformó en organización nacional y 2 años mas tarde se fundaba la American Society of Children's Dentistry, apareciendo en 1928 la primera revista científica dedicada a la odontología pediátrica, el actual Journal of Dentistry for Children.

En el siglo XVIII nace el padre de la odontopediatría moderna, Robert Bunon (1702-1748). Este autor francés fija las grandes líneas del estudio sobre la prevención y la odontología conservadora, sus demostraciones y teorías sobre la odontología infantil son originales y merecen nuestra atención. Nacido en el pueblo francés de Garnison, en 1735, llega a París realizando el examen de cirujano-dentista en San Cosme e interesándose en particular por las hipoplasias del esmalte $^{33}$. En 1924 se crea el primer texto "Odontología para niños" del autor Hogeboom. Otros clásicos como Choen, Fin, McDonald, Barber, Pinkham han sido guías para el aprendizaje de la odontología pediátrica.

\section{La odontología mexicana}

La medicina, de muy alto nivel, que se desarrolló en el México prehispánico, incluía el conocimiento y la atención de problemas dentales. Se practicaban también desgastes dentales e incrustaciones de piedras semipreciosas por motivos ornamentales. Durante el virreinato, fueron los barberos flebotomianos los encargados de realizar extracciones a las personas que sufrían de dolores dentales. A principio del siglo XIX, empezaron a llegar a México, dentistas franceses y norteamericanos que dieron a conocer esta novedosa profesión, hasta entonces desconocida en nuestro país. La sociedad Mexicana acogió con beneplácito a estos personajes que realizaban toda clase de tratamientos dentales como extracciones, orificaciones, puentes y hermosas dentaduras de caucho y vulcanita. Muy pronto algunos jóvenes mexicanos fueron aprendiendo las "Artes Dentales", en los gabinetes de los dentistas extranjeros, para después titularse como cirujanos dentistas de la Escuela Nacional de Medicina, a partir de la inauguración del Consultorio Nacional de Enseñanza Dental, primera escuela de odontología del país, que al paso del tiempo se transformaría en la Facultad de Odontología de la Universidad Nacional Autónoma de México. ${ }^{34,35}$

Sobre la fundación de los primeros posgrados en odontología pediátrica no se tiene información precisa, pero la demanda de atención bucal en niños ha aumentado y con ello las instituciones especializadas en la formación de especialistas en el área.

El objetivo de este artículo es informar tanto a egresados de la carrera de odontología, odontopediatras e instituciones que se encargan de certificar la calidad de posgrados, la distribución de las universidades que cuentan en su currícula 
con el posgrado o maestría de odontología pediátrica en la república y su estatus de certificación.

REGIÓN NOROESTE (1 posgrado).

1. Universidad Autónoma de Baja California (UABC) Baja California: Tijuana ${ }^{2}$

REGIÓN NORTE (5 posgrados).

2. Universidad Autónoma de Coahuila (UAC) Coahuila: Torreón ${ }^{3}$

3. Universidad Autónoma de Ciudad Juárez (UACJ) Chihuahua: Cd. Juárez ${ }^{5}$

4. Universidad Autónoma de Chihuahua Chihuahua: Chihuahua ${ }^{6}$

5. Universidad Autónoma de Nuevo León (UANL) Nuevo León: Monterrey y San Nicolás de los Garza ${ }^{21}$

6. Universidad Autónoma de Tamaulipas (UAT) Tamaulipas: Tampico ${ }^{28}$

REGIÓN BAJÍO (5 posgrados)

7. Instituto Latinoamericano de Ciencias y Humanidades Guanajuato: Guanajuato ${ }^{14}$

8. Universidad de la Salle Bajío A.C. Guanajuato: León ${ }^{13}$

9. Universidad Autónoma de Querétaro (UAQ) Querétaro: Querétaro ${ }^{25}$

10. Universidad Autónoma de San Luis Potosí (UASLP) San Luis Potosí: San Luis Potosí ${ }^{26}$

11. Universidad Autónoma de Zacatecas (UAZ) "Francisco García Salinas" Zacatecas: Guadalupe ${ }^{32}$

\section{REGIÓN CENTRO Y GOLFO (7 posgrados)}

12. Universidad Latinoamericana (ULA) Morelos: Cuernavaca ${ }^{18}$
13. Universidad Autónoma Benito Juárez de Oaxaca (UABJO) Oaxaca: Oaxaca ${ }^{22}$

14. Benemérita Universidad Autónoma de Puebla (BUAP) Puebla: Puebla ${ }^{23}$

15. Hospital para el Niño Poblano (HNP) Puebla: Puebla ${ }^{24}$

16. Universidad Veracruzana (UV) Veracruz: Xalapa $^{30}$

17. Universidad Juárez Autónoma de Tabasco (UJAT) Tabasco: Villahermosa ${ }^{27}$

18. Universidad Autónoma de Tlaxcala (UAT) Tlaxcala: Tlaxcala ${ }^{29}$

REGIÓN SURESTE (1 posgrado)

19. Universidad Autónoma de Yucatán (UADY) Yucatán: Mérida ${ }^{31}$

REGIÓN MÉXICO D.F. (6 posgrados)

20. Universidad Nacional Autónoma de México (UNAM) México DF: Copilco ${ }^{7}$

21. Facultad de Estudios Superiores Zaragoza (UNAM) México DF: Iztapalapa ${ }^{7}$

22. Universidad Tecnológica de México (UNITEC) México DF: Miguel Hidalgo ${ }^{8}$

23. Hospital Infantil de México “Federico Gómez" México DF ${ }^{10}$

24. Instituto Nacional de Pediatría (INP) México $\mathrm{DF}^{12}$

25. Universidad Autónoma del Estado de México (UAEM) Estado de México: Toluca9

\section{REGIÓN OCCIDENTE (5 posgrados)}

26. Universidad de Guadalajara (U.DE.G) Jalisco: Guadalajara ${ }^{15}$ 
27. Universidad Autónoma de Guadalajara (UAG) Jalisco: Guadalajara ${ }^{16}$

28. Universidad Cuauhtémoc plantel Guadalajara Jalisco: Guadalajara ${ }^{17}$
29. Centro de Estudios de Posgrados en Odontología (CEPO) Jalisco: Guadalajara ${ }^{20}$

30. Universidad Autónoma de Nayarit (UAN) Nayarit: Tepic ${ }^{19}$

Tabla 1. Universidades con posgrado en odontopediatría

\begin{tabular}{|c|c|c|c|c|}
\hline UNIVERSIDAD & ESTADO & CAMPUS & DURACIÓN & GRADO \\
\hline $\begin{array}{l}\text { 1. Universidad Autónoma de Baja California } \\
\text { (UABC) }\end{array}$ & $\begin{array}{c}\text { Baja } \\
\text { California }\end{array}$ & Tijuana & 2 años & Especialidad \\
\hline 2. Universidad Autónoma de Coahuila (UAC) & Coahuila & Torreón & 2 años & Maestría \\
\hline 3. Universidad Autónoma de Ciudad Juárez (UACJ) & Chihuahua & Cd. Juárez & 2 años & Especialidad \\
\hline 4. Universidad Autónoma de Chihuahua & Chihuahua & Chihuahua & & Maestría \\
\hline $\begin{array}{l}\text { 5. Universidad Nacional Autónoma de México } \\
\text { (UNAM) }\end{array}$ & México DF & Copilco & 2 años & Especialidad \\
\hline $\begin{array}{l}\text { 6. Facultad de Estudios Superiores Zaragoza } \\
\text { (UNAM) }\end{array}$ & México DF & Iztapalapa & 2 años & Especialidad \\
\hline 7. Universidad Tecnológica de México (UNITEC) & México DF & $\begin{array}{l}\text { Miguel } \\
\text { Hidalgo }\end{array}$ & 2 años & Especialidad \\
\hline $\begin{array}{l}\text { 8. Universidad Autónoma del Estado de México } \\
\text { (UAEM) }\end{array}$ & $\begin{array}{l}\text { Estado de } \\
\text { México }\end{array}$ & Toluca & 2 años & Especialidad \\
\hline Hospital Infantil de México "Federico Gómez" & México DF & México DF & 2 años & Especialidad \\
\hline Instituto Nacional de Pediatría (INP) & México DF & México DF & 2 años & Especialidad \\
\hline 9. Universidad de la Salle Bajío A.C. & Guanajuato & León & 3 años $1 / 2$ & Maestria \\
\hline $\begin{array}{l}\text { 10. Instituto Latinoamericano de Ciencias y } \\
\text { Humanidades }\end{array}$ & Guanajuato & Guanajuato & 2 años & Especialidad \\
\hline 11. Universidad de Guadalajara (U.DE.G) & Jalisco & Guadalajara & 2 años & Especialidad \\
\hline 12. Universidad Autónoma de Guadalajara (UAG) & Jalisco & Guadalajara & 2 años & Especialidad \\
\hline 13. Universidad Cuauhtémoc plantel Guadalajara & Jalisco & Guadalajara & 2 años & Maestría \\
\hline 14. Universidad Latinoamericana (ULA) & Morelos & Cuernavaca & 2 años & Especialidad \\
\hline 15. Universidad Autónoma de Nayarit (UAN) & Nayarit & Tepic & 2.5 años & Especialidad \\
\hline $\begin{array}{l}\text { Centro de Estudios de Posgrados en Odontología } \\
\text { (CEPO) }\end{array}$ & Jalisco & Guadalajara & 3 años & Especialidad \\
\hline 16. Universidad Autónoma de Nuevo León (UANL) & Nuevo León & $\begin{array}{l}\text { Monterrey y } \\
\text { San Nicolás } \\
\text { de los Garza }\end{array}$ & 2.5 años & Maestría \\
\hline $\begin{array}{l}\text { 17. Universidad Autónoma Benito Juárez de Oaxaca } \\
\text { (UABJO) }\end{array}$ & Oaxaca & Oaxaca & 2 años & Maestría \\
\hline $\begin{array}{l}\text { 18. Benemérita Universidad Autónoma de Puebla } \\
\text { (BUAP) }\end{array}$ & Puebla & Puebla & 3 años & Maestría \\
\hline
\end{tabular}




\begin{tabular}{|c|c|c|c|c|}
\hline $\begin{array}{l}\text { Hospital para el Niño Poblano } 2 \text { años especialidad } \\
\text { (HNP) }\end{array}$ & Puebla & Puebla & 2 años & Especialidad \\
\hline 19. Universidad Autónoma de Querétaro (UAQ) & Querétaro & Querétaro & 2 años & Especialidad \\
\hline $\begin{array}{l}\text { 20. Universidad Autónoma de San Luis Potosí } \\
\text { (UASLP) }\end{array}$ & $\begin{array}{l}\text { San Luis } \\
\text { Potosí }\end{array}$ & $\begin{array}{l}\text { San Luis Po- } \\
\text { tosí }\end{array}$ & 2 años & Especialidad \\
\hline $\begin{array}{l}\text { 21. Universidad Juárez Autónoma de Tabasco } \\
\text { (UJAT) }\end{array}$ & Tabasco & Villahermosa & 2 años & Especialidad \\
\hline 22. Universidad Autónoma de Tamaulipas (UAT) & Tamaulipas & Tampico & 2 años & Especialidad \\
\hline 23. Universidad Autónoma de Tlaxcala (UAT) & Tlaxcala & Tlaxcala & 2 años & Especialidad \\
\hline 24. Universidad Veracruzana (UV) & Veracruz & Xalapa & 2 años & Especialidad \\
\hline 25. Universidad Autónoma de Yucatán (UADY) & Yucatán & Mérida & 2 años & Maestría \\
\hline $\begin{array}{l}\text { 26. Universidad Autónoma de Zacatecas (UAZ) } \\
\text { "Francisco García Salinas" }\end{array}$ & Zacatecas & Guadalupe & & Especialidad \\
\hline
\end{tabular}

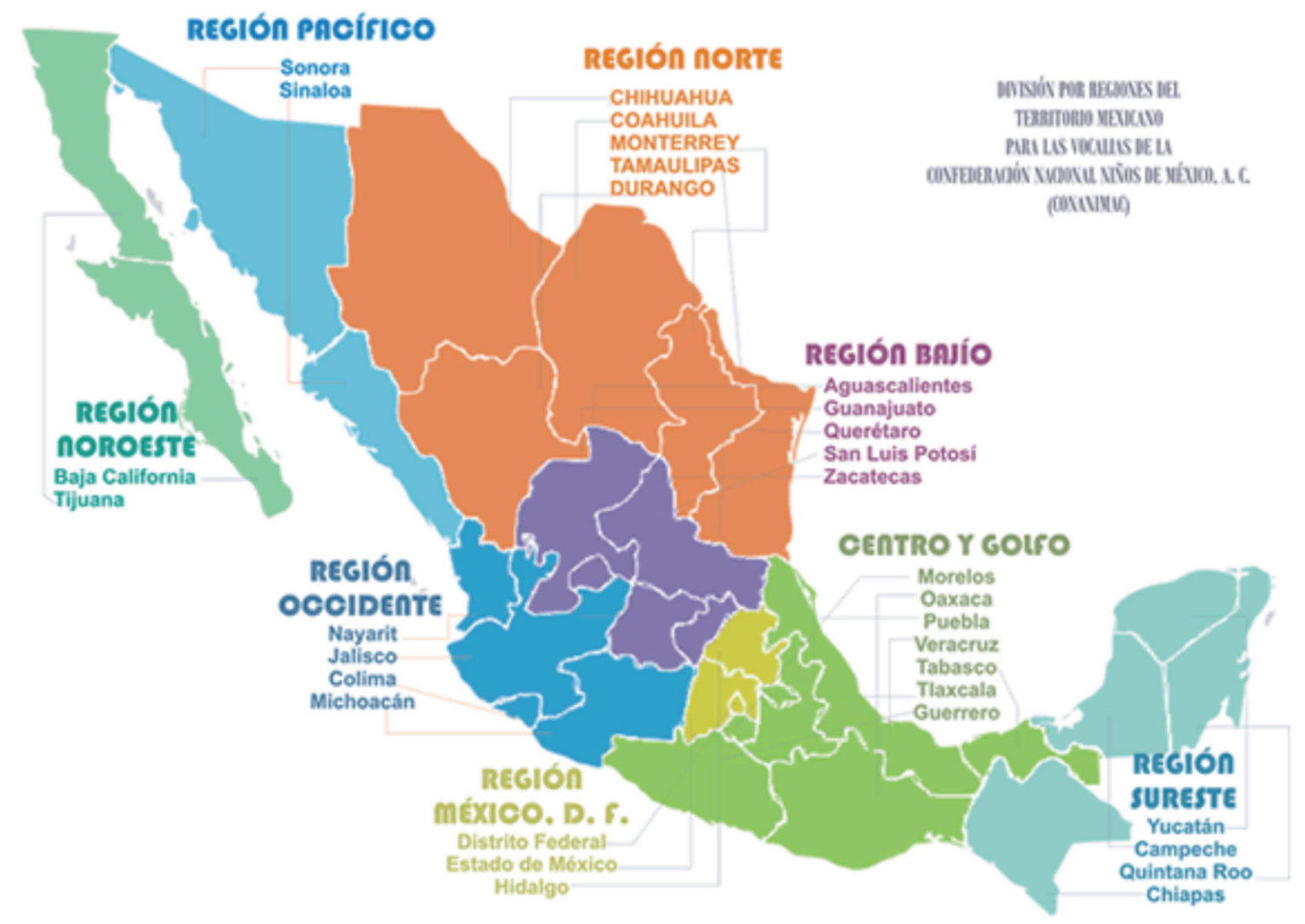

Figura 1. Regiones de la República Méxicana.

\section{Discusión}

Los datos demográficos arrojan que la población total nacional es de 112.336.538 donde solo 72.514.513 cuentan con servicios de salud y ma- yor número de personal médico, en primer lugar la región México DF sin contar Hidalgo, en la región centro y bajío se encuentra el estado de Veracruz en cuarto lugar y Puebla en séptimo lugar, el estado de Oaxaca en décimo lugar, los 
Tabla 2. Población de 18 años y más con estudios de posgrado.

\begin{tabular}{|c|c|c|}
\hline $\begin{array}{c}\text { Población } \mathbf{> 1 8} \text { años de edad } \\
\text { con estudios de posgrado }\end{array}$ & Región & $\begin{array}{c}\text { No. De posgrados de } \\
\text { Odontopediatría }\end{array}$ \\
\hline Distrito Federal & México DF & 5 \\
\hline México & México DF & 1 \\
\hline Jalisco & Occidente & 4 \\
\hline Nuevo León & Norte & 1 \\
\hline Veracruz & Centro y Golfo & 1 \\
\hline Puebla & Centro y Golfo & 2 \\
\hline Guanajuato & Bajío & 2 \\
\hline Chihuahua & Norte & 2 \\
\hline Baja California & Noroeste & 1 \\
\hline Sonora & Pacífico & 0 \\
\hline
\end{tabular}

Fuente: 36

estados restantes no entran dentro de los 10 primeros con servicios médicos. Tlaxcala, Morelos, Hidalgo, Guerrero y Tabasco son de los últimos lugares en servicios de salud y personal médi$\mathrm{CO}^{36}$.

En cuanto a la población con estudios de posgrado de 18 años y más (Tabla 2), la zona sureste, occidente y noroeste son las que cuentan con menor cantidad de posgrados. Sin embargo la zona del pacífico se encuentra totalmente descuidada sin instituciones de educación superior con posgrados de odontopediatría, aunque sea una de las 10 más importantes en el desarrollo de otros posgrados. ${ }^{36}$

De acuerdo a los datos demográficos ${ }^{36}$, la población total nacional es de 112.336.538 habitantes, en la REGIÓN CENTRO Y GOLFO existe la mayor concentración de posgrados en odontopediatria (7 posgrados) que tiene una relación directa con el número total de habitantes en los estados que conforman dicha región:
Total Región Centro: 22.410.751 habitantes:

- Estado de Morelos: 1.777.227 habitantes.

- Estado de Oaxaca: 3.801.962 habitantes.

- Estado de Puebla: 5.779.829 habitantes.

- Estado de Veracruz: 7.643.194 habitantes.

- Estado de Tabasco: 2.238.603 habitantes.

- Estado de Tlaxcala: 1.169.936 habitantes.

En la REGIÓN MÉXICO D.F. existen 6 posgrados para un total de 8.851.080 habitantes que conforman la región.

De acuerdo a los datos anteriores se concluye que hay correspondencia en el número de instituciones de educación superior con posgrados de odontopediatría y el número de habitantes que conforman la región centro y región México $\mathrm{DF}$, sin embargo los estados que tienen un mayor número de habitantes de 0 a 14 años (29.3\% total 
de la población) son los estados de Chiapas (región suroeste) y Guerrero (región centro golfo) los cuales no cuentan con posgrados de odontopediatría; de los 32 estados el Distrito Federal y Nuevo León cuentan con el menor número de niños de 0 a 14 años y son parte de las regiones donde se encuentra la mayor concentración de instituciones de educación superior y posgrados en odontopediatría. Chiapas y Guerrero por su bajo nivel de desarrollo en instituciones de educación superior y posgrados no cuentan con la especialidad y es donde existe el mayor número de población de 0 a 14 años de edad por lo tanto mayor demanda de odontopediatras.

Es importante destacar que pueden existir universidades no afiliadas a los institutos de educación de los gobiernos de los estados no reconocidas y actualmente se encuentran formando especialistas de baja calidad, aumentando el número de egresados del área y con ello disminuyendo las oportunidades de trabajo en regiones donde existe menor concentración de población infantil. Se sugiere estandarizar la acreditación de programas teóricos y clínicos y que la capación del personal docente sea de calidad.

\section{Conclusiones}

De las regiones de la República Mexicana (Fig. 1) en las que se encuentran ubicados los diversos posgrados, se concluye que de un total de 30 universidades que cuentan con la especialidad o maestría en odontopediatría, existen 7 en la denominada región centro y golfo y 6 en la región México D.F., estos datos concuerdan con el elevado número de habitantes que habitan esas regiones mas no con el número de población infantil que requiera sus servicios debido a que los estados con mayor población de 0 a 14 años son Chiapas y Guerrero lugares donde por su bajo desarrollo en educación superior no existen posgrados que formen odontopediatras.

\section{Referencias}

1. Academia Mexicana de Odontología Pediátrica [en línea]. [actualizado 2008; accesado 27 Feb 2012] Disponible en: http: / / amop.org.mx/ programas.html

2. Universidad Autónoma de Baja California [en línea]. [ actualizado 2007 ; accesado 27 Feb 2012] Disponible en: http: / / www.uabc.mx/

3. Universidad Autónoma de Coahuila [en línea]. [actualizado 2012; accesado 27 Feb 2012] Disponible en: http: / / www. postgradoeinvestigacion.uadec.mx / posgrados / PNPC / M-cienciasodontologicas / objetivo.html

4. F.M.F.E.O. Federación Mexicana de Facultades y Escuelas de Odontología [en línea]. [actualizado 2011; accesado 27 Feb 2012] Disponible en: http://www.fmfeo.org.mx/index.php?option=com_content\&view=article\&id=64\&Item id $=141$

5. Universidad Autónoma de Ciudad Juárez [en línea]. [accesado 27 Feb 2012] Disponible en: http:/ / www.uacj.mx

6. Universidad Autónoma de Chihuahua [en línea]. [actualizado 2008; accesado 27 Feb 2012] Disponible en: http:// www.eo.uach.mx/posgradoindex.php

7. Universidad Nacional Autónoma de México. [en línea]. [actualizado 2012; accesado 27 Feb 2012] Disponible en: http:// www.odonto.unam.mx/ http://74.53.185.66/ odonto/pdfs/odontopediatria.pdf http://www.zaragoza. unam.mx/

8. Universidad Tecnológica de México. [en línea]. [actualizado 2010; accesado 27 Feb 2012] Disponible en: http:/ / www. unitec.mx/odontologia-pediatrica\# 
9. Universidad Autónoma del Estado de México. [en línea]. [accesado 27 Feb 2012] Disponible en: www.uaemex.mx/ fodontologia/

10. Hospital Infantil de México “Federico Gómez" [en línea]. [actualizado 2010; accesado 27 Feb 2012] Disponible en: http: / / www.himfg.edu.mx/

11. Subsecretaría de Innovación y Calidad. Dirección General de Calidad y Educación en Salud. Dirección de Estomatología. Dirección Médica del ISSSTE. [en línea]. [actualizado 2009; accesado 27 Feb 2012] Disponible en: http:/ / www. calidad.salud.gob.mx/doctos/educacion/convo_mere2010_2011.pdf

12. Instituto Nacional de Pediatría [en línea]. [actualizado 2012; accesado 27 Feb 2012] Disponible en: http: / / www.pediatria.gob.mx/interior8.html

13. Universidad De La Salle Bajío [en línea]. [actualizado 2011; accesado 27 Feb 2012] Disponible en: http:/ / bajio.delasalle.edu.mx/oferta/mae_odo_pediatrica.php

14. Instituto Latinoamericano de Ciencias y Humanidades [en línea]. [actualizado 2010; accesado 27 Feb 2012] Disponible en: www.ilaleon.com

15. Universidad de Guadalajara. Centro Universitario de Ciencias de la Salud [en línea]. [actualizado 2010; accesado 27 Feb 2012] Disponible en: www.cucs.udg.mx/odontologia

16. Universidad Autónoma de Guadalajara [en línea]. [accesado 27 Feb 2012] Disponible en: http: / / www.uag.mx / index. php?option=com_content\&view=article\&id=134\&Itemid=194\&lang=mx

17. Universidad Cuauhtémoc [en línea]. [actualizado 2011; accesado 27 de febrero 2012] Disponible en: http:// www.cuauhtemocgdl.com.mx/ site/index.php?option=com_content\&view=article\&id=219:maestria-enodontopediatria\&catid=99:pos-ciencias-de-la-salud\&Itemid=133

18. Universidad Latinoamericana Nayarit [en línea]. [actualizado 2011; accesado 27 de febrero 2012] Disponible en: http:/ / www.ula.edu.mx/index.php/especialidades/odontopediatria

19. Universidad Autónoma de Nayarit [en línea]. [actualizado 2011; accesado 27 de febrero 2012] Disponible en: http: / / www.uan.edu.mx/es/especialidad-en-odontopediatria\#perfil_

20. Centro de Estudios de Posgrado en Odontopediatría [en línea]. [actualizado 2012; accesado 27 de febrero 2012] Disponible en: http: / / www.cepomexico.com.mx/ver.asp?id=19

21. Universidad Autónoma de Nuevo León [en línea]. [actualizado 2012; accesado 27 de febrero 2012] Disponible en: http: / / www.uanl.mx / oferta / maestria-en-ciencias-odontologicas-con-orientacion-en-odontopediatria.html

22. Universidad Autónoma "Benito Juárez" de Oaxaca [en línea]. [ accesado 27 de febrero 2012] Disponible en: www. uabjo.mx http:/ / www.uabjo.mx/oferta/maestria/odontologiaEspecialidadClinica.pdf

23. Benemérita Universidad Autónoma de Puebla [en línea]. [ accesado 27 de febrero 2012] Disponible en: http: / / www. posgradoestomatologia.buap.mx/

24. Hospital para el niño Poblano [en línea]. [actualizado 2012; accesado 27 de febrero 2012] Disponible en: www.hnp. org.mx

25. Universidad Autónoma de Querétaro [en línea]. [actualizado 2012; accesado 27 de febrero 2012] Disponible en: http: / / www.uaq.mx/academico/posgrados/

26. Universidad Autónoma de San Luis Potosí [en línea]. [actualizado 2012; accesado 27 de febrero 2012] Disponible en: http: / / www.uaslp.mx/Spanish/Paginas/ default.aspx

27. Universidad Juárez Autónoma de Tabasco [en línea].[actualizado 2012; accesado 27 de febrero 2012] Disponible en: http: / / www.ujat.mx/

28. Universidad Autónoma de Tamaulipas [en línea]. [actualizado julio2012; accesado 27 de febrero 2012] Disponible en: http: / / rectoria.uat.edu.mx/investigacionyposgrado/default.asp. http://rectoria.uat.edu.mx/investigacionyposgrado/posgrado/posgrados_2011.pdf

29. Universidad Autónoma de Tlaxcala [en línea]. [actualizado 2003; accesado 27 de febrero 2012] Disponible en: http: / / www.uatx.mx/oferta_academica/index.php?programa=60\&plan=1353

30. Universidad Veracruzana [en línea]. [actualizado 2011; accesado 27 de febrero 2012] Disponible en: http://www. uv.mx/eoi/ 
31. Facultad de Odontología de la Universidad Autónoma de Yucatán [en línea]. [actualizado2010; accesado27 de febrero 2012] Disponible en: http:/ / www.odontologia.uady.mx/info_gral/odontopediatria.php

32. Universidad Autónoma de Zacatecas “Francisco García Salinas" [en línea]. [accesado 27 de febrero 2012] Disponible en: http: / / 148.217.18.210/ ofertaeducativa/ view / ofertaEducativa.php?idPrograma=144010\&idUnidad=22200

33. Boj JR, García BC, Mendoza A, Catalá M. Odontopediatría. 1ª ed. 2004. 544 págs. Editorial: ELSEVIER-MASSON

34. McCracken. Prótesis Parcial Removible. 10ª ed. Editorial Medica Panamericana 2000.

35. Stewart, Rudd. Prostodoncia parcial removible. $2^{\mathrm{a}}$ ed. Actualidades medico odontologicas latinoamericana, CaracasVenezuela. 1992.

36. INSTITUTO NACIONAL DE ESTADISTICA, GEOGRAFÍA E INFORMÁTICA. [en línea] [accesado 14 de noviembre 2012] Disponible en: http:/ / www.inegi.gob.mx /

Recibido: 16-08-2012

Aceptado: 25-12-2012

Correspondencia: hutizilnet@yahoo.com 